\title{
O calendário do povo Palikur-Arukwayene ${ }^{1}$
}

\author{
Adonias Guiome Ioiôn
}

DOI: http://dx.doi.org/10.20435/tellus.v19i38.623

O calendário do meu povo Palikur-Arukwayene, sempre segue os sinais e a orientação da natureza para deliberar o período do início e do final do ano. 0 ano, no nosso território divide-se duas estações designadas digiswiki/muwokwekri (estação das chuvas/inverno) que se inicia no mês que os não indígenas chamam de dezembro e termina no final de junho3; ; e kamukwiki/abareswan (estação da seca/ verão) entre os meses de julho e novembro. Nas duas temporadas, os períodos são definidos pelos vegetais, animais, peixes e aves abundantes no período, mas se utiliza também a orientação da lua e das constelações para o desenvolvimento das atividades. As estações são essenciais à sobrevivência do meu povo que observa o seu próprio calendário, diferente do calendário dos não indígenas, conforme o nosso modo de vida e a nossa cultura. Mas, também existem outras maneiras de marcar o tempo, por exemplo, por intermédio das festas tradicionais, dos cantos de animais e aves, os quais se associam ao início do período de derrubada, queima, coivara e colocação das roças e, finalmente a colheita; a alteração do nível das águas do igarapé, do rio e do campo indica a possibilidade de caça de alguns animais e aves, favorece a pesca e ainda a coleta de frutos.

\footnotetext{
${ }^{1}$ As informações que eu tinha sobre o calendário do meu povo foi complementado e traduzido da língua Parikwaki pelo Aldiere Orlando Arukwayene do clã Wayveyene nos dias 28 e 29 de dezembro de 2018, pois a tradução requer muitos cuidados. Aldiere é Licenciado Pleno em Letras, pela Universidade Estadual Vale do Acaraú e também trabalhou com a Diana Green, como tradutor em língua Parikwaki há muitos anos, quando o Summer Institut of Linguistic se instalou entre nós. Venho colhendo dados para escrever sobre este calendário desde o ano de 2013, com o senhor Uwetmin (Manoel Antônio dos Santos), Sidronio Labontê do clã kawakyene, José Corréio do clã wayveyene e Zilar dos Santos arukwayano, os nossos sábios. No mês de julho do ano de 2017, conversei com o senhor Motye (Manoel José loiô) e com colaboradores Denildo Labontê, Lucilene Guiome loiô e Jackson Guiome loiô, sobre alguns períodos referentes aos peixes e às plantações.

2 Universidade Federal do Pará (UFPA), Belém, Pará, Brasil.

${ }^{3}$ Usarei no texto as marcações do calendário pertencente aos não indígenas, o denominado calendário Gregoriano, assim facilito a vida do leitor.
} 
O povo Palikur-Arukwayene divide o ano em quatro estações ou em duas. Os períodos destas duas maneiras de distinguir as estações não coincidem complementarmente. As quatro estações são denominadas de acordo com os quatro níveis d'água no pantanal ao redor das ilhas em que vivemos. As estações são: digiswiki "enchente", un-ad "água-zão", nimehme "vazante", e kamuw-kri "sol-estação".

A outra maneira de dividir o ano é em duas estações: a de chuva (denominada muwokwe-kri "chuva-estação") com início a partir do mês de dezembro e a de seca (abereswan "verão") que inicia a partir do mês de julho, chegando a novembro. Cada chuva é também denominada pelo nome da constelação que pode ser vista durante o período daquela chuva: kayeb, tavara, kusuvwi eggutye, kusuvwi isamwitye e wayam. Dizemos que as estrelas "chamam" as chuvas. As histórias tradicionais relacionam as chuvas com a posição dos astros, e assim o povo se orienta para o plantio e a colheita para poder se alimentar. É importante lembrar que cada estação, período ou tempo serve, ainda, para a recordar, por exemplo, do nascimento das crianças ou de tempos importantes relativos às festas e às celebrações, como me ensinou Aldiere Orlando.

Agora, apresento as correlações feitas, por nós, em função da marcação do tempo Palikur-Arukwayene, nos quadros abaixo. Indico as chuvas, como marcador es do tempo porque, no Oiapoque, onde moramos elas são importantes, sua chegada anuncia um período com possibilidade de fartura alimentar obtida com muito trabalho. 
Quadros - Os meses dos Palikur-Arukwayene e os períodos de cada um dos vegetais, animais, peixes e aves, com o respectivo guia das constelações

\begin{tabular}{|c|c|}
\hline $\begin{array}{l}\text { 1. KAYEB-DEZEMBRO } \\
\text { (início da 1ạ chuva-estação) }\end{array}$ & $\begin{array}{c}\text { KAYEB-JANEIRO } \\
\text { (continua 1a chuva-estação) }\end{array}$ \\
\hline $\begin{array}{c}\text { Ahawkanavrik pitatye muwokwe-kri } \\
\text { "chuva-estação" in kavusaw giwntak } \\
\text { warukma Kayeb, datka, adahan akavuska } \\
\text { digiswiki hawata adahan ibekhene } \\
\text { kamukri, ig padak aynesa un. } \\
\text { Início da fase da 1ạ chuva-estação para } \\
\text { "enchente", que divide o ano, vem da } \\
\text { constelação denominada de Kayeb, a } \\
\text { serpente, e inicia com a chuva leve e com } \\
\text { pouca água. }\end{array}$ & $\begin{array}{l}\text { Tiviknene ahawkanavrik pitatye } \\
\text { muwokwekri Kayeb, adahan digiswiki, im } \\
\text { turukwiyos. } \\
\text { Continua a fase da 1ạ chuva-estação } \\
\text { do Kayeb para "enchente", com chuva } \\
\text { moderada e leve, período em que os } \\
\text { peixes começam a adentrar o campo } \\
\text { alagado para a reprodução. }\end{array}$ \\
\hline $\begin{array}{l}\text { Ahawkanavrik was akewkanavrik hawata } \\
\text { mutuhka amutribdi. } \\
\text { Período do cultivo da roça/plantação de } \\
\text { mandiocas e suas diversas espécies. }\end{array}$ & $\begin{array}{l}\text { Tiviknene ahawkanavrik was akewkanavrik } \\
\text { hawata mutuhka amutribdi. } \\
\text { Continua o período do cultivo da roça/ } \\
\text { plantação de mandiocas e suas diversas } \\
\text { espécies. }\end{array}$ \\
\hline $\begin{array}{c}\text { Ahawkanavrik mutuhka amutribdi: } \\
\text { pilatnu, kayg, kawar. } \\
\text { Início da época da plantação: das } \\
\text { bananas, batata doce, abacaxi, e outros } \\
\text { cultivos. }\end{array}$ & $\begin{array}{l}\text { Tiviknene ahawkanavrik mutuhka } \\
\text { amutribdi: pilatnu, kayg, kawar. } \\
\text { Continua a época da plantação: das } \\
\text { bananas, batata doce, abacaxi, etc. }\end{array}$ \\
\hline $\begin{array}{l}\text { Ahawkanavrik wokiwka } \\
\text { Época da bacaba. }\end{array}$ & $\begin{array}{l}\text { Tiviknene hawkanavrik wokiwka. } \\
\text { Ahawkanavrik mutuhka uwas. } \\
\text { Continua época da bacaba, e plantação } \\
\text { de laranjas. }\end{array}$ \\
\hline $\begin{array}{l}\text { Ahawkanavrik kariwru } \\
\text { Época de tamuatá. }\end{array}$ & $\begin{array}{l}\text { Ahawkanavrik mutuhka tukugu. } \\
\text { Tempo da plantação da cabaça. }\end{array}$ \\
\hline $\begin{array}{l}\text { Ahawkanavrik kariwyanewka } \\
\text { Época de ovos de tamuatás. }\end{array}$ & $\begin{array}{l}\text { Ahawkanavrik ivuriti bekwevye. } \\
\text { Tempo de florescimento das árvores e das } \\
\text { plantas. }\end{array}$ \\
\hline $\begin{array}{l}\text { Ahawkanavrik hagawyan. } \\
\text { Época da desova do peixe limpa-vidro. }\end{array}$ & $\begin{array}{l}\text { Ahawkanavrik kariwyan bakwa. } \\
\text { Tempo da reprodução dos ovos de } \\
\text { tamuatás. }\end{array}$ \\
\hline $\begin{array}{l}\text { Ahawkanavrik pune akak iyg. } \\
\text { Época do peixe jeju e do peixe traíra. }\end{array}$ & $\begin{array}{l}\text { Ahawkanavrik kunan bekwevye } \\
\text { Tempo da reprodução dos ovos de } \\
\text { tucunarés. }\end{array}$ \\
\hline
\end{tabular}




\begin{tabular}{|c|c|}
\hline $\begin{array}{c}\text { 1. KAYEB-DEZEMBRO } \\
\text { (início da 1a chuva-estação) }\end{array}$ & $\begin{array}{c}\text { KAYEB-JANEIRO } \\
\text { (continua 1ä chuva-estação) }\end{array}$ \\
\hline $\begin{array}{c}\text { Ahawkanavrik umayan. } \\
\text { Época da piranha. }\end{array}$ & $\begin{array}{c}\text { Ahawkanavrik umayan bekwevye } \\
\text { Tempo da reprodução dos ovos das } \\
\text { piranhas. }\end{array}$ \\
\hline $\begin{array}{c}\text { Ahawkanavrik kunanewka } \\
\text { Época de tucunarés. }\end{array}$ & $\begin{array}{c}\text { Ahawkanavrik iyg bekwevye } \\
\text { Tempo da reprodução dos ovos das } \\
\text { traíras. }\end{array}$ \\
\hline $\begin{array}{c}\text { Ahawkanavrik pareyne, punamna akak } \\
\text { mewka. }\end{array}$ & $\begin{array}{c}\text { Ahawkanavrik im ber, duway akak wakay } \\
\text { kanpiyos. }\end{array}$ \\
\hline Época de jacaré e tracajá. & Tempo da desova dos peixes cará. \\
\hline & $\begin{array}{c}\text { Ahawkanavrik umayan bekwevye. } \\
\text { Época da reprodução dos ovos da } \\
\text { piranha. }\end{array}$ \\
\hline & $\begin{array}{c}\text { Ahawkanavrik uvayan axwevyos. } \\
\text { Época dos patos que comem nos campos } \\
\text { secos. }\end{array}$ \\
\hline
\end{tabular}

\begin{tabular}{|c|c|}
\hline $\begin{array}{l}\text { 2. TAVARA-FEVEREIRO } \\
\text { (2a chuva-estação) }\end{array}$ & $\begin{array}{l}\text { WAKTI-MARÇO } \\
\text { (3a chuva-estação) }\end{array}$ \\
\hline $\begin{array}{l}\text { Ahawkanavrik aveynivye muwokwekri } \\
\text { in kavusaw giwntak warukma Tavara, } \\
\text { kuhivra, "un-ad" kuwis, henneme hawata } \\
\text { ig padak aynesa un. } \\
\text { Início da fase da 2a chuva-estação } \\
\text { vem da constelação de astro Martim- } \\
\text { Pescador, o pássaro, época em que o } \\
\text { nível d'água "água-zão" no pantanal já é } \\
\text { razoavelmente elevada ao redor das ilhas. }\end{array}$ & $\begin{array}{c}\text { Ahawkanavrik amamnampiye } \\
\text { muwokwekri in kavusaw giwntak } \\
\text { warukma Wakti, adahanwata digiswiki, } \\
\text { hawata ig padak aynesa un. } \\
\text { Início da fase da 3ạ chuva-estação vem } \\
\text { da constelação de astro Wakti, as sete } \\
\text { estrelas, também época em o nível d’água } \\
\text { no pantanal continua razoavelmente } \\
\text { elevada ao redor das ilhas. }\end{array}$ \\
\hline $\begin{array}{c}\text { Ahawkanavrik mewka turukwiyo. } \\
\text { Época de tracajás. }\end{array}$ & $\begin{array}{l}\text { Ahawkanavrik matiska kavunma. } \\
\text { Tempo da safra de maracujá. }\end{array}$ \\
\hline $\begin{array}{l}\text { Ahawkanavrik punamna } \\
\text { Época de jacarés. }\end{array}$ & $\begin{array}{l}\text { Ahawkanavrik hiyeg kanikne muwapuw } \\
\text { kariymadga. } \\
\text { Início da época da pescaria no campo } \\
\text { alagado, como: acará-apaiari, piranha, } \\
\text { tucunaré, acará, et.) }\end{array}$ \\
\hline $\begin{array}{c}\text { Ahawkanavrik waratwewka } \\
\text { Época dos tucumãs. }\end{array}$ & $\begin{array}{l}\text { Ahawkanavrik kamaxka mewka akak } \\
\text { punamna kariymadga. } \\
\text { Época da pesca do tracajá e jacaré no } \\
\text { campo alagado. }\end{array}$ \\
\hline
\end{tabular}




\begin{tabular}{|c|c|}
\hline $\begin{array}{c}\text { 2. TAVARA-FEVEREIRO } \\
\text { (2a chuva-estação) }\end{array}$ & $\begin{array}{c}\text { WAKTI-MARÇO } \\
\text { (3a chuva-estação) }\end{array}$ \\
\hline $\begin{array}{c}\text { Ahawkanavrik im parekwiye kariymadga. } \\
\text { Época dos peixes quando entram no } \\
\text { campo alagado, como: tucunaré, piranha, } \\
\text { danché, jeju, traira, acará-apaiari, et. }\end{array}$ & $\begin{array}{c}\text { Ahawkanavrik wewvaki. } \\
\text { Época da caçada. }\end{array}$ \\
\hline $\begin{array}{c}\text { Tiviknene ahawkanavrik kewka was. } \\
\text { Continua o período do cultivo da roça/ } \\
\text { plantação de mandiocas. }\end{array}$ & $\begin{array}{c}\text { Akavuska ahawkanavrik waraka kaneg ku } \\
\text { pariye mutuhka kamukri mpiyatinene. } \\
\text { Início da época da safra de mandioca do } \\
\text { cultivo do ano anterior. }\end{array}$ \\
\hline $\begin{array}{c}\text { Amaksemni ahawkanavrik mutuhka pilatnu. } \\
\text { Término da época da plantação das } \\
\text { bananas variadas. }\end{array}$ & \\
\hline
\end{tabular}

\begin{tabular}{|c|c|}
\hline $\begin{array}{l}\text { 3. KUSUVWI-EGGUTYE-ABRIL } \\
\text { (4ª chuva-estação) }\end{array}$ & $\begin{array}{l}\text { KUSUVWI-ISAMWITYE-MAIO } \\
\text { (5a chuva-estação) }\end{array}$ \\
\hline $\begin{array}{l}\text { Ahawkanavrik avaxnikanpiye muwokwekri } \\
\text { in kavusaw giwntak warukma Kusuvwi- } \\
\text { Eggutye, ner Kusuvwi padak kiyeste un. } \\
\text { Início da fase da 4a chuva-estação } \\
\text { vem da constelação de astro Kusuvwi- } \\
\text { Eggutye, o irmão mais velho, sendo nesta } \\
\text { época o nível d'água no pantanal já é } \\
\text { consideravelmente elevada em relação as } \\
\text { estações anteriores, mas não tão alto. }\end{array}$ & $\begin{array}{c}\text { Ahawkanavrik avohwoknipiye } \\
\text { muwokwekri in kavusaw giwntak } \\
\text { warukma Kusuvwi-Isamwitye. Ner } \\
\text { Kusuvwi padak ka aynsima un, keh hiyeg } \\
\text { danuh muwapuw amadga umuh. } \\
\text { Início da fase da 5a chuva-estação vem da } \\
\text { constelação de astro Kusuvwi-lsamwitye, } \\
\text { o irmão mais novo, mas este astro é a } \\
\text { continuação fase da 4a chuva-estação } \\
\text { e tem chuva muito forte e demorada, e } \\
\text { nessa época o nível d'água no pantanal } \\
\text { chega ao máximo. E é nessa época que } \\
\text { todos conseguem chegar nos lugares mais } \\
\text { distantes da região por meio da canoa. }\end{array}$ \\
\hline $\begin{array}{l}\text { Akavuska ahawkanavrik wayabrewka } \\
\text { Início da época dos peixes danché. }\end{array}$ & $\begin{array}{l}\text { Ahawkanavrik wayabrewka } \\
\text { Época dos peixes danché }\end{array}$ \\
\hline $\begin{array}{l}\text { Ahawkanavrik sawaygewka. } \\
\text { Época do peixe piaba subindo o rio em } \\
\text { grandes números. }\end{array}$ & $\begin{array}{c}\text { Ahawkanavrik kuwekweyanewka } \\
\text { Época dos filhotes dos papagaios (quando } \\
\text { são procurados e criados). }\end{array}$ \\
\hline $\begin{array}{l}\text { Ahawkanavrik duway, umayan, kunan, } \\
\text { wayabra. } \\
\text { Época do peixe acará-apaiari, piranha, } \\
\text { tucunaré, danché, etc. }\end{array}$ & $\begin{array}{l}\text { Ahawkanavrik duway, umayan, kunan, } \\
\text { wayabra. } \\
\text { Época do peixe acará-apaiari, piranha, } \\
\text { tucunaré, danché, etc. }\end{array}$ \\
\hline
\end{tabular}




\begin{tabular}{|c|c|}
\hline $\begin{array}{l}\text { 3. KUSUVWI-EGGUTYE-ABRIL } \\
\text { (4ạ chuva-estação) }\end{array}$ & $\begin{array}{l}\text { KUSUVWI-ISAMWITYE-MAIO } \\
\text { (5a chuva-estação) }\end{array}$ \\
\hline $\begin{array}{l}\text { Akavuska ahawkanavrik kehka umuh. } \\
\text { Início da época da produção de canoas } \\
\text { pequenos, médios e grandes. }\end{array}$ & $\begin{array}{l}\text { Ahawkanavrik kehka umuh. } \\
\text { Época da produção de canoas pequenos, } \\
\text { médios e grandes. }\end{array}$ \\
\hline $\begin{array}{l}\text { Ahawkanavrik isaw } \\
\text { Época de frutos de buriti. }\end{array}$ & $\begin{array}{l}\text { Ahawkanavrik isaw } \\
\text { Época de frutos de buriti. }\end{array}$ \\
\hline $\begin{array}{c}\text { Ahawkanavrik was higapka. } \\
\text { Época do açaí. }\end{array}$ & $\begin{array}{c}\text { Ahawkanavrik was higapka. } \\
\text { Época do açaí. }\end{array}$ \\
\hline \multirow[t]{5}{*}{$\begin{array}{c}\text { Ahawkanavrik payt kehka. } \\
\text { Tempo para construção de casa. }\end{array}$} & $\begin{array}{c}\text { Ahawkanavrik payt kehka. } \\
\text { Tempo para construção de casa. }\end{array}$ \\
\hline & $\begin{array}{l}\text { Ahawkanavrik makananu. } \\
\text { Época do "makananu" maduros, tipo de } \\
\text { cipó que dá frutas unidas e tem gosto de } \\
\text { gelatinas. }\end{array}$ \\
\hline & $\begin{array}{l}\text { Ahawkanavrik karikti. } \\
\text { Época do inajá. }\end{array}$ \\
\hline & $\begin{array}{l}\text { Ahawkanavrik wiwka isaw adahan pah. } \\
\text { Tempo para tirar pé de buriti para servir } \\
\text { de ponte entre ilhas no tempo da seca. }\end{array}$ \\
\hline & $\begin{array}{l}\text { Ahawkanavrik tivigu. } \\
\text { Tempo de andiroba. }\end{array}$ \\
\hline
\end{tabular}

\begin{tabular}{|c|c|}
\hline $\begin{array}{c}\text { 4. WASAWKA-JUNHO } \\
\text { (tempo da chuva fraca) }\end{array}$ & $\begin{array}{c}\text { IHUKWAKI/ABERESWAN-JULHO } \\
\text { (estação da seca) }\end{array}$ \\
\hline $\begin{array}{c}\text { Akavuska ahawkanavrik wasawka. } \\
\text { Início do período da roçagem de roças } \\
\text { (preparação). }\end{array}$ & $\begin{array}{c}\text { Akavuska abereswan, hawata un kavusaw } \\
\text { makar. Muwok wayk pahaymine. } \\
\text { Início da estação da seca e ou "vazante". } \\
\text { Nesta época o nível d'água começa a } \\
\text { baixar e a chuva é passageira. }\end{array}$ \\
\hline $\begin{array}{c}\text { Ahawkanavrik was puhiviyo. } \\
\text { Época da safra do açaí. }\end{array}$ & $\begin{array}{c}\text { Akavuska ihukwaki was. } \\
\text { Início da época de derrubada da roça. }\end{array}$ \\
\hline $\begin{array}{c}\text { Akavuska kuwekweyanawka. } \\
\text { Início da época dos papagaios. }\end{array}$ & $\begin{array}{c}\text { Ahawkanavrik abereswan. } \\
\text { Época do início de verão. }\end{array}$ \\
\hline Ahawkanavrik karikti ivat. & $\begin{array}{c}\text { Ahawkanavrik kuwa } \\
\text { Período de caranguejos. }\end{array}$ \\
\hline & $\begin{array}{c}\text { Ahawkanavrik avatiyavrik amutri: siku, } \\
\text { kayg, kawar, kavar. }\end{array}$ \\
& $\begin{array}{c}\text { Início da época das plantas maduras: } \\
\text { cana-de-açúcar, batata, abacaxi e cará). }\end{array}$ \\
\hline
\end{tabular}




\begin{tabular}{|c|c|}
\hline $\begin{array}{l}\text { 4. WASAWKA-JUNHO } \\
\text { (tempo da chuva fraca) }\end{array}$ & $\begin{array}{l}\text { IHUKWAKI/ABERESWAN-JULHO } \\
\text { (estação da seca) }\end{array}$ \\
\hline & $\begin{array}{l}\text { Ahawkanavrik kuwekwe, yawk, surusru, } \\
\text { maras, arawa, arudikavra, maremre. } \\
\text { Época dos papagaios, tucanos, sururinas, } \\
\text { jacu, araras, maitaca-roxa, jandaia } \\
\text { (quando começam as travessias em } \\
\text { grande número para outras ilhas) } \\
\end{array}$ \\
\hline & $\begin{array}{l}\text { Ahawkanavrik miyumyu. } \\
\text { Época do ingá }\end{array}$ \\
\hline & $\begin{array}{c}\text { Ahawkanavrik inuvra. } \\
\text { Início da época da fruta do pepino-do- } \\
\text { mato. }\end{array}$ \\
\hline & $\begin{array}{l}\text { Ahawkanvrik karikti ivat. } \\
\text { Época do inajá. }\end{array}$ \\
\hline & $\begin{array}{l}\text { Ahawkanavrik tukugu ivat. } \\
\text { Tempo da cabaça madura. }\end{array}$ \\
\hline & $\begin{array}{l}\text { Ahawkanavrik kehka iyakokti: imedgit akak } \\
\text { yakot adahan havisne im. } \\
\text { Tempo de confeçãa de armas como: } \\
\text { arcos e flechas para flechar peixes no rio. }\end{array}$ \\
\hline & $\begin{array}{l}\text { Ahawkanavrik ahayak anunu. } \\
\text { Tempo do mel de abelha. }\end{array}$ \\
\hline
\end{tabular}

\begin{tabular}{|c|c|}
\hline $\begin{array}{c}\text { 5. WAYAM-AGOSTO } \\
\text { (6a chuva-estação do Wayam) }\end{array}$ & $\begin{array}{c}\text { IWANYANEWKA-SETEMBRO } \\
\text { (início da desova dos animais répteis/ } \\
\text { estação da seca) }\end{array}$ \\
\hline $\begin{array}{l}\text { Ahawkanavrik avuguhkunpiye hawata } \\
\text { amaksemni muwokwekri in kavusaw } \\
\text { giwntak warukma Wayam, ner wayk } \\
\text { adahan makarasene un, mmanawa } \\
\text { giwaykni mpiya kibehtenwa. } \\
\text { Início da fase da 6ạ chuva-estação vem } \\
\text { da constelação de astro Wayam, o jabuti, } \\
\text { sendo que esta última época considerada } \\
\text { como estação da chuva-vazante, pois } \\
\text { nessa estação a chuva é passageira e os } \\
\text { campos vão secando. }\end{array}$ & $\begin{array}{l}\text { Ahawkanavrik iwanyanewka. Um tiviknene } \\
\text { kehwenene. } \\
\text { Época da desova de camaleão-fêmea. A } \\
\text { água no campo continua diminuindo. }\end{array}$ \\
\hline
\end{tabular}




\begin{tabular}{|c|c|}
\hline $\begin{array}{l}\text { 5. WAYAM-AGOSTO } \\
\text { (6ª chuva-estação do Wayam) }\end{array}$ & $\begin{array}{c}\text { IWANYANEWKA-SETEMBRO } \\
\text { (início da desova dos animais répteis/ } \\
\text { estação da seca) }\end{array}$ \\
\hline $\begin{array}{c}\text { Akavuska ahawkanavrik kunan } \\
\text { axnumewviyos. } \\
\text { Início da época dos tucunarés, quando } \\
\text { flutuam no rio. }\end{array}$ & $\begin{array}{l}\text { Akavuska mewkanyanewka. } \\
\text { Início do tempo dos ovos de tracajá. }\end{array}$ \\
\hline $\begin{array}{c}\text { Época de derrubada da roça (nas áreas de } \\
\text { capoeira ou na mata virgem). }\end{array}$ & $\begin{array}{c}\text { Ahawkanavrik kunan axnumewviyos. } \\
\text { Época dos tucunarés, quando boiam no } \\
\text { rio. }\end{array}$ \\
\hline $\begin{array}{l}\text { Ahawkanavrik ahiwka amutribdi ariw. } \\
\text { Início da época da safra de frutos. }\end{array}$ & $\begin{array}{c}\text { Ahawkanavrik wayabra axnumewviyes } \\
\text { Época dos peixes danchés, quando boiam } \\
\text { no rio. }\end{array}$ \\
\hline $\begin{array}{c}\text { Ahawkanavrik inuvra ivat. } \\
\text { Época da fruta do pepino do mato. }\end{array}$ & $\begin{array}{l}\text { Ahawkanavrik iwanyanewka. } \\
\text { Época da desova de camaleão-fêmea. }\end{array}$ \\
\hline \multirow[t]{6}{*}{$\begin{array}{l}\text { Hawkanavrik amutibdi ivatviyos: xiku, } \\
\text { kayg, kawar, parasu e kavar. } \\
\text { Início da época plantas maduras: cana- } \\
\text { de- acurçar, batata, abacaxi, parasu e } \\
\text { cará. }\end{array}$} & $\begin{array}{c}\text { Hawata ahawkanavrik sig awna adahan } \\
\text { ekkene amaksemni tirahka was amun } \\
\text { wasewnibdi. } \\
\text { Também época do som de lagartos } \\
\text { pequenos para anunciar que está } \\
\text { chegando ao fim do período de derrubada } \\
\text { de roças nas capoeiras. } \\
\end{array}$ \\
\hline & $\begin{array}{c}\text { Ahawkanavrik sig awna, parakwa awna } \\
\text { adahan ekkene abereswan. } \\
\text { Época do cântico da cigarra, do jacu para } \\
\text { anunciar a época do verão/seca. }\end{array}$ \\
\hline & $\begin{array}{l}\text { Ahawkanavrik was wasewni tigahka akak } \\
\text { ihukwaki. } \\
\text { Época da roçagem de roças pequenas e } \\
\text { derrubações das roças. }\end{array}$ \\
\hline & $\begin{array}{l}\text { Ahawkanavrik uwas ivat. } \\
\text { Época das laranjas maduras. }\end{array}$ \\
\hline & $\begin{array}{l}\text { Ahawkanavrik bayag. } \\
\text { Tempo de aruanãs. }\end{array}$ \\
\hline & $\begin{array}{l}\text { Ahawkanavrik kuwik avuri bakwa. } \\
\text { Tempo de florescimento do ipê. }\end{array}$ \\
\hline
\end{tabular}




\begin{tabular}{|c|c|}
\hline $\begin{array}{l}\text { 6. MEWKAYANEWKA / OUTUBRO } \\
\text { (Kamuw-kri / sol-estação) }\end{array}$ & $\begin{array}{l}\text { WAS AKEWKANAVRIK / NOVEMBRO } \\
\text { (Kamuw-kri / sol-estação) }\end{array}$ \\
\hline $\begin{array}{l}\text { Amaksemni ahawkanavrik areketni } \\
\text { kamukri, abet ner kayg ka awahnisima } \\
\text { kamuw, kariymadga kaba makar. } \\
\text { Última estação que divide o ano é } \\
\text { conhecido como sol-estão, período } \\
\text { quente, e o campo está quase seca. }\end{array}$ & $\begin{array}{l}\text { Kamuw tiviknene ka awahnisima hawata } \\
\text { kariymadga kuwis makar. Hiyeg danuh ta } \\
\text { pawkat aminew pah. } \\
\text { Continua o período do sol-estação e o } \\
\text { campo já está seca. As pessoas chegam } \\
\text { em suas roças, caminhando em cima dos } \\
\text { buritizeiros. }\end{array}$ \\
\hline $\begin{array}{c}\text { Ahawkanavrik mewkanyanewka. } \\
\text { Época dos ovos do tracajá. }\end{array}$ & $\begin{array}{l}\text { Ahawkanavrik was kewka, mutuhka } \\
\text { amutribdi: daxin, kavar, siku, kayg, kawar } \\
\text { e parasu. } \\
\text { Início do período do cultivo da roça/ } \\
\text { plantação de mandiocas, daxin, cará, } \\
\text { cana-de-açucar, bata doce, abacaxi e } \\
\text { cará-espinhosa. }\end{array}$ \\
\hline $\begin{array}{l}\text { Ahawkanavrik abereswan } \\
\text { Período do verão. }\end{array}$ & $\begin{array}{l}\text { Akavuska ahawkanavrik wokiwka. } \\
\text { Início da época da bacaba. }\end{array}$ \\
\hline $\begin{array}{l}\text { Ahawkanavrik was bukahka } \\
\text { Período da queimada de roça. }\end{array}$ & $\begin{array}{c}\text { Akavuska ahawkanavrik hagawyan. } \\
\text { Início da época dos ovos do peixe limpa- } \\
\text { vidro. }\end{array}$ \\
\hline $\begin{array}{l}\text { Ahawkanavrik ikevwiyiki. } \\
\text { Época de coivara da roça. }\end{array}$ & $\begin{array}{l}\text { Ahawkanavrik parakwa awna adahan } \\
\text { ekkene akavuska muwokwekri. } \\
\text { Época dos cânticos do pássaro aracuã- } \\
\text { de-cabeça-vermelha, significa que está } \\
\text { chegando a época da chuva. }\end{array}$ \\
\hline $\begin{array}{c}\text { Akavuska tivuw awna adahan ekkene } \\
\text { kennesa ahawkanavrik muwokwekri. } \\
\text { Início do cântico do sapo, isso significa que } \\
\text { está chegando o período da chuva. }\end{array}$ & $\begin{array}{l}\text { Ahawkanavrik bayag. } \\
\text { Tempo de aruanãs. }\end{array}$ \\
\hline $\begin{array}{l}\text { Ahawkanavrik mank } \\
\text { Época da manga. }\end{array}$ & $\begin{array}{c}\text { Ahawkanavrik kariwru, pareyne, mewka } \\
\text { ku pariye misakwa anavi timisne } \\
\text { abet abereswan, kennesa akigbimna } \\
\text { mahakwa akak parewni. } \\
\text { Época de tamuatá, jacaré e tracajá que } \\
\text { ficam em baixo dos barros no verão } \\
\text { nos campos, próximos dos lagos e nos } \\
\text { igarapés). }\end{array}$ \\
\hline
\end{tabular}




\begin{tabular}{|c|c|}
\hline $\begin{array}{l}\text { 6. MEWKAYANEWKA / OUTUBRO } \\
\text { (Kamuw-kri / sol-estação) }\end{array}$ & $\begin{array}{l}\text { WAS AKEWKANAVRIK / NOVEMBRO } \\
\text { (Kamuw-kri / sol-estação) }\end{array}$ \\
\hline $\begin{array}{l}\text { Ahawkanavrik tukwa. } \\
\text { Tempo da galega. }\end{array}$ & $\begin{array}{c}\text { Ahawkanavrik kayka aramtem abet kayg } \\
\text { ameremni. } \\
\text { Época da Dança/Festa do Turé na lua } \\
\text { cheia. }\end{array}$ \\
\hline $\begin{array}{c}\text { Ahawkanavrik kuhivrebdi warikavrikya akak } \\
\text { amadgaya mahakwa: daga, waratna, wakar, } \\
\text { uvayan, masiwgu, agaykwa akak durukwa. } \\
\text { Tempo dos pássaros que existem no rio e } \\
\text { nos lagos como: maguari, garça, arapapá, } \\
\text { pato, marreco, biguatinga, mergulhão, } \\
\text { socó-boi, etc. } \\
\end{array}$ & \\
\hline $\begin{array}{c}\text { Ahawkanavrik bayagewka. } \\
\text { Tempo de aruanãs. }\end{array}$ & \\
\hline $\begin{array}{c}\text { Ahawkanavrik im timis avigku warik: kunan, } \\
\text { ber, umayan, iyg, wayabra, waygu, wakay, } \\
\text { xugutub akak masug. } \\
\text { Tempo dos peixes que flutuam no rio } \\
\text { como: tucunaré, cará, piranha, traíra, } \\
\text { danché, pratinha, acará, peixe boca-de- } \\
\text { fogo, acará-açu, etc. } \\
\end{array}$ & \\
\hline $\begin{array}{l}\text { Ahawkanavrik mihitwi. } \\
\text { Tempo de caju. }\end{array}$ & \\
\hline $\begin{array}{c}\text { Ahawkanavrik mayaksaki/axwaki avawyewa } \\
\text { ariw paytwempu avigku warik Arukwa. } \\
\text { Tempo de feriado ou descanso/tempo para } \\
\text { piquenique/tempo para os arukwayene } \\
\text { ir com toda a família fora da comunidade } \\
\text { a procura de alimentos ao longo rio } \\
\text { Urucauá. } \\
\end{array}$ & \\
\hline $\begin{array}{c}\text { Ahawkanavrik kehka kuwak, hiyeg } \\
\text { misakwa/himak aynte pawka awaku } \\
\text { aviyukan was. } \\
\text { Tempo para fabricar farinha na roça, mas } \\
\text { dormem na casa da farinha devido a seca e } \\
\text { a distância da roça. }\end{array}$ & \\
\hline $\begin{array}{l}\text { Ahawkanavrik pilatnu ivat. } \\
\text { Tempo da banana madura. }\end{array}$ & \\
\hline
\end{tabular}


Para o meu povo o ano se inicia no mês de dezembro, ou seja, quando o kayeb começa a derramar água sobre nós, fazendo chover de acordo com a época. As chuvas de alguma forma comandam o ritmo da vida, pois elas favorecem o aparecimento, o plantio, a caça, a pesca e a coleta deste ou daquele produto que se transforma em alimento para nós os Palikur-Arukwayene.

\section{Sobre o autor:}

Adonias Guiome Ioiô: Doutorando em Antropologia na Universidade Federal do Pará (UFPA). Mestre em Antropologia junto ao Programa de Pós-Graduação em Antropologia (PPGA/UFPA). Professor de Língua e Cultura Indígena na Escola Indígena Estadual Moisés laparrá, no Oiapoque-Amapá e pesquisador do Núcleo Kusurwi de Estudos Palikur/Arukwayene (NUKEPA). Bolsista da CAPES: E-mail: adoniasarukwayene@gmail.com

Recebido em: 11 de fevereiro de 2019

Aprovado em: 11 de março de 2019 
